Pesq. Vet. Bras. 30(10):881-886, outubro 2010

\title{
Anatomia do plexo braquial de macaco-barrigudo (Lagothrix lagothricha) ${ }^{1}$
}

\begin{abstract}
Gessica Ariane M. Cruz ${ }^{2}$ e Marta Adami ${ }^{3 *}$
ABSTRACT.- Cruz G.A.M. \& Adami M. 2010. [Anatomy of the brachial plexus of the Woolly-Monkey (Lagothrix lagothricha.] Anatomia do plexo braquial de macaco-barrigudo (Lagothrix lagothricha). Pesquisa Veterinária Brasileira 30(10):881-886. Departamento de Anatomia dos Animais Domésticos, Escola de Medicina Veterinária, Universidade Federal da Bahia, Av. Adhemar de Barros 500, Ondina, Salvador, BA 40170-110, Brazil. E-mail: madami@ufba.br

The woolly-monkey (Lagothrix lagothricha) is an antropoid belonging to the Atelidae Family which includes the largest neotropical primates. A female cadaver woolly-monkey was fixed in a 10\% formaldehyde solution and dissected using a stereoscopic magnifying glass and photodocumented. The brachial plexus originated from the spinal nerves $\mathrm{C} 5$ to $\mathrm{C} 8$ and T1, forming the cranial, medium, and caudal stems, from which derived the peripheral nerves; those nerves had similar origin and innervation area when compared to plexuses from other primates, with the exception of the musculocutaneous nerve that crossed the coracobraquial muscle. Data from studies with brachial plexus from primates allow the access to valuable information regarding the morphology of those animals, and could also assist in the establishment of anatomical parameters among species, which could then contribute to anesthetic procedures and injury treatments.
\end{abstract}

INDEX TERMS: Nervous plexus, nervous system, Lagothrix lagothricha.

RESUMO.- O macaco-barrigudo (Lagothrix lagothricha) é um antropóide pertencente à Família Atelidae que possui os maiores primatas neotropicais. Um cadáver fêmea de macaco-barrigudo foi fixado com solução de formaldeído a $10 \%$, posteriormente dissecado com o auxílio de lupa estereoscópica e fotodocumentado. O plexo braquial originou-se dos nervos espinhais $\mathrm{C} 5$ a C8 e T1, formando os troncos cranial, médio e caudal, dos quais derivaram os nervos periféricos que se assemelharam na origem e no território de inervação com os plexos de outros primatas, com exceção do nervo musculocutâneo que atravessou o músculo coracobraquial. Pesquisas sobre o plexo braquial de primatas fornecem dados que disponibilizam o acesso a informações valiosas sobre a morfologia destes animais e auxiliam no estabelecimento de parâmetros anatômicos

\footnotetext{
${ }^{1}$ Recebido em 1 der março de 2010.

Aceito para publicação em 5 de agosto de 2010.

2 Curso de Medicina Veterinária, Escola de Medicina Veterinária, Universidade Federal da Bahia (UFBA), Salvador, BA, Brasil. E-mail: aririvet@gmail.com

${ }^{3}$ Departamento de Anatomia dos Animais Domésticos, Escola de Medicina Veterinária, UFBA, Avenida Adhemar de Barros 500, Campus Universitário de Ondina, Salvador, BA 40170-110. *Autor para correspondência: madami@ufba.br
}

entre as espécies, contribuindo também no tratamento de injúrias e procedimentos anestésicos.

TERMOS DE INDEXAÇÃO: Plexo braquial, sistema nervoso, Lagothrix lagothricha.

\section{INTRODUÇÃO}

O macaco-barrigudo (Lagothrix lagothricha Humboldt, 1812) encontra-se distribuído desde o Brasil até a Colômbia (Napier \& Napier 1967, Chiarelli 1972, Hill 1972, Auricchio 1995, Reis et al. 2006). Vivem em grupos com indivíduos do mesmo gênero ou associados a outros primatas em florestas primárias com até $3.000 \mathrm{~m}$ de altitude, possuem habilidade de semibraquiação relacionada com sua dieta basicamente frugívora (Napier \& Napier 1967, Auricchio 1995, Reis et al. 2006). Atualmente sua situação é de "baixo risco" pela IUCN (International Union for Conservation of Nature and Natural Resources), porém até 1994 seu estado era vulnerável (Rylands 2007).

O plexo braquial de primatas tem sido amplamente estudado em espécies de Macaca em analogia com o plexo braquial do homem (Brooks 1883, Sugiyama 1965), em gorila (Gorilla sp.), chimpanzé (Pan sp.), orangotango (Pongo sp.) e gibão (Hylobates sp.) (Hepburn 1892, Oliveira 2003), 
gálagos (Galago senegalensis senegalensis) (Kanagasuntheram \& Mahran 1960), macaco-de-cheiro (Saimiri sciureus) (Mizuno 1969a), macaco-prego-de-cara-branca (Cebus capucinus) (Mizuno 1969b), chimpanzés e micos (Troglotydes niger e Cynocephalus anubis) (Champneys 1975), babuínos (Papio ursinus) (Booth et al. 1997), macaco-prego (Cebus apella) (Ribeiro 2002).

Dados sobre as características morfológicas de macaco-barrigudo ainda são escassos e destacam-se estudos sobre análises funcionais de estruturas relacionadas à postura e locomoção (Ziemer 1978, Johnson \& Shapiro 1998, Youlatos 2000). Hill (1972) descreveu sobre o esqueleto, trato digestório e órgão dos sentidos.

Objetivou-se descrever o plexo braquial de Lagothrix lagothricha em sua origem, composição, nervos derivados e território de inervação, ressaltando a importância do conhecimento anatômico como base para o estabelecimento de parâmetros anatômicos entre as espécies e também contribuir no tratamento de injúrias e nos procedimentos anestésicos.

\section{MATERIAL E MÉTODOS}

O macaco-barrigudo (Lagothrix lagothricha) é um primata pertencente à Infraordem Platyrrhini, que abrange os macacos do novo mundo e faz parte da Família Atelidae que possui os maiores primatas neotropicais e Gênero Lagothrix (Napier \& Napier 1967, Chiarelli 1972). Um exemplar fêmea que veio a óbito por causas naturais, foi cedido pelo Planeta Zoo, situado no município de Lauro de Freitas, Bahia, para o Departamento de Anatomia dos Animais Domésticos da Escola de Medicina Veterinária, Universidade Federal da Bahia (UFBA). A pesquisa foi autorizada pelo Sistema de Autorização e Informação em Biodiversidade (SISBIO) do Instituto Brasileiro do Meio Ambiente e dos Recursos Naturais Renováveis (IBAMA) com o no 17907-1 em 18.11.2008. O animal foi fixado com solução de formaldeído a $10 \%$ injetada via artéria carótida comum e posteriormente, os dois antímeros foram dissecados com o auxílio de lupa estereoscópica (PZOLabimex) e fotodocumentados. A terminologia adotada foi baseada no International Committee on Veterinary Gross Anatomical Nomenclature (2005).

\section{RESULTADOS}

O macaco-barrigudo apresentou sete vértebras cervicais, com a emergência do primeiro par de nervos espinhais entre o occipital e o atlas. O plexo braquial originou-se a partir do quinto, sexto, sétimo e oitavo segmentos medulares cervicais e primeiro segmento medular torácico, que corresponderam às vértebras cervicais $5^{\circ}, 6^{\circ}, 7^{\circ} \mathrm{e}$ $8^{\circ}$ e à $1^{\text {a }}$ vértebra torácica. Os nervos espinhais $\mathrm{C} 5 \mathrm{a}$ C8 e T1 formaram três troncos: cranial (C5 e C6), médio (C7) e caudal (C8 e T1).

Em ambos os antímeros observou-se a alça axilar formada pela artéria axilar e o nervo mediano. Os troncos cranial e médio originaram sete nervos cada um e o tronco caudal seis. Foram eles: tronco cranial: nervo supraescapular, nervo subescapular, nervo musculocutâneo, nervo mediano, nervo radial, nervo axilar e nervo toracodorsal, sendo dois exclusivos do tronco: nervo supraescapular e nervo musculocutâneo. Tronco médio: nervo subescapular, nervo peitoral, nervo mediano, nervo radial, nervo axilar, nervo torácico longo, nervo toracodorsal e apenas um restrito ao tronco: nervo torácico longo. Tronco caudal: nervo mediano, nervo ulnar, nervo radial, nervo toracodorsal, nervo cutâneo medial do antebraço e dois exclusivos do tronco: nervo ulnar e nervo cutâneo medial do antebraço.

Ramos comunicantes entre os nervos foram observados entre os nervos musculocutâneo e mediano. O NMC emitiu um ramo comunicante para o NM no terço proximal do braço, o que implicou a contribuição do tronco cranial na formação do NM. No terço distal do mesmo segmento o NMC recebeu um ramo comunicante do NM. (Quadro 1, Fig.1 e 2)

Quadro 1. Plexo braquial de macaco-barrigudo (Lagothrix lagothricha): nervos, origem e território de inervação

\begin{tabular}{|c|c|c|}
\hline Nervo & Origem & Território de inervação \\
\hline Supraescapular (NSP) & Tronco cranial & $\begin{array}{l}\text { Músculo deltóide, músculo supraespinhoso } \\
\text { e músculo infraespinhoso }\end{array}$ \\
\hline Subescapulares (NnSB) & Troncos cranial e médio & Músculo subescapular e músculo redondo maior \\
\hline Peitorais $(\mathrm{NnP})$ & C6 e tronco médio & Músculos peitorais \\
\hline Musculocutâneo (NMC) ${ }^{\mathrm{a}}$ & Tronco cranial & $\begin{array}{l}\text { Músculo coracobraquial, supre as duas porções } \\
\text { do músculo } \\
\text { bíceps braquial e músculo braquial }\end{array}$ \\
\hline Mediano (NM) & Todos os troncos & $\begin{array}{l}\text { Músculo braquial, porção curta do músculo bíceps } \\
\text { braquial e porção média do músculo tríceps braquial }\end{array}$ \\
\hline Ulnar (NU) & Tronco caudal & Porção média do músculo tríceps braquial \\
\hline Radial (NR) & Todos os troncos & $\begin{array}{l}\text { Todas porções do músculo tríceps braquial, múscu- } \\
\text { lo braquial }\end{array}$ \\
\hline Axilar (NA) & Troncos cranial e médio & $\begin{array}{l}\text { Músculo redondo maior, músculo redondo menor e } \\
\text { músculo deltóide }\end{array}$ \\
\hline Torácico longo (NTL) & Tronco médio & Músculo serrátil ventral do tórax \\
\hline Toracodorsal (NTD) & Todos os troncos & Músculo grande dorsal \\
\hline $\begin{array}{l}\text { Cutâneo medial do } \\
\text { antebraço (NCMA) }\end{array}$ & Tronco caudal & Fáscia do antebraço \\
\hline
\end{tabular}

a Atravessou o músculo coracobraquial. 


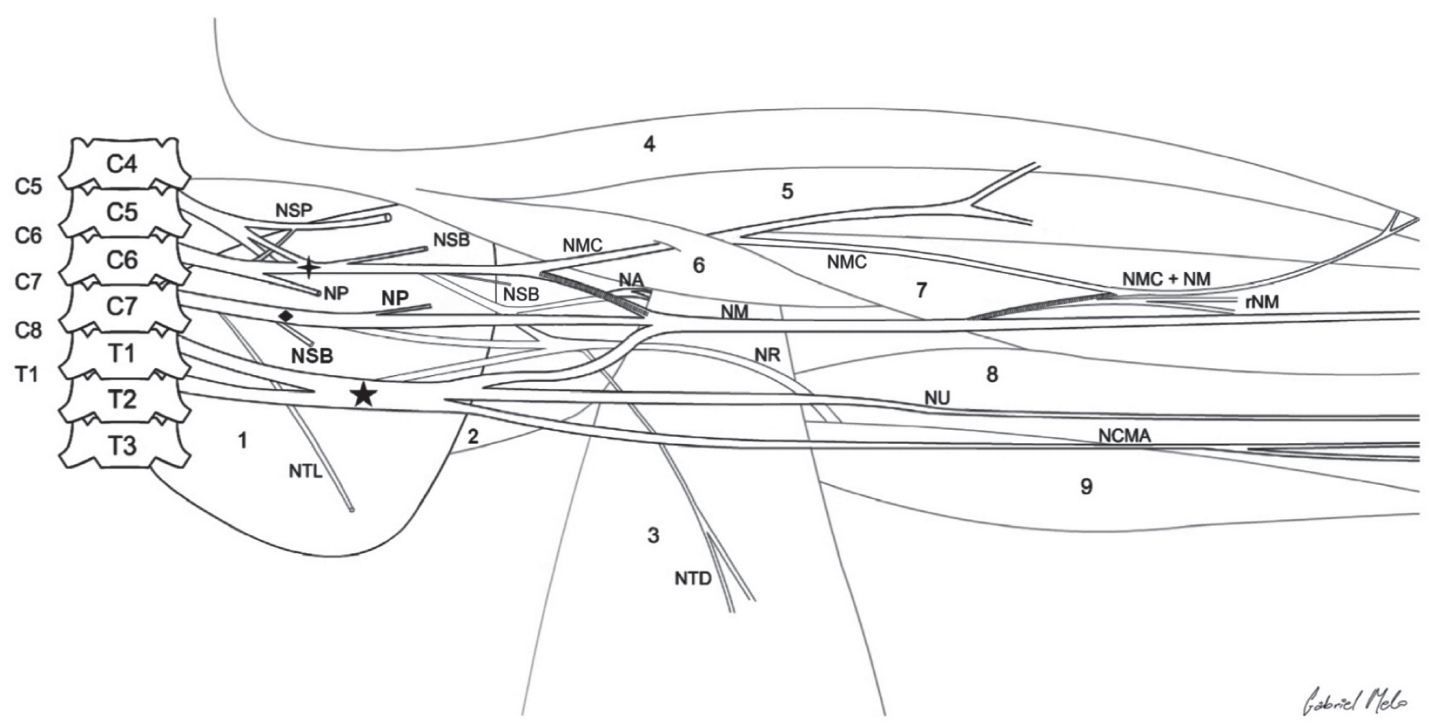

Fig.1. Desenho esquemático com representação da origem, composição e território de inervação do plexo braquial esquerdo de macaco-barrigudo (Lagothrix lagothricha). C5 a C8: quinto a oitavo nervos espinhais cervicais; T1: primeiro nervo espinhal torácico. Troncos cranial $(+)$, médio $(\diamond)$ e caudal ( $\star$ ). Músculos-(1) subescapular; (2) redondo maior; (3) grande dorsal; (4 e 5): bíceps braquial; 6-coracobraquial; (7) braquial; (8 e 9) tríceps braquial. Nervos: NSP: supraescapular; NSB: subescapular; NP: peitorais; NTL: torácico longo; NA: axilar; NM: mediano; NMC: musculocutâneo; NR: radial; NTD: toracodorsal; NU: ulnar; NCMA: cutâneo medial do antebraço. Ramos comunicantes do NMC com o NM: áreas sombreadas.

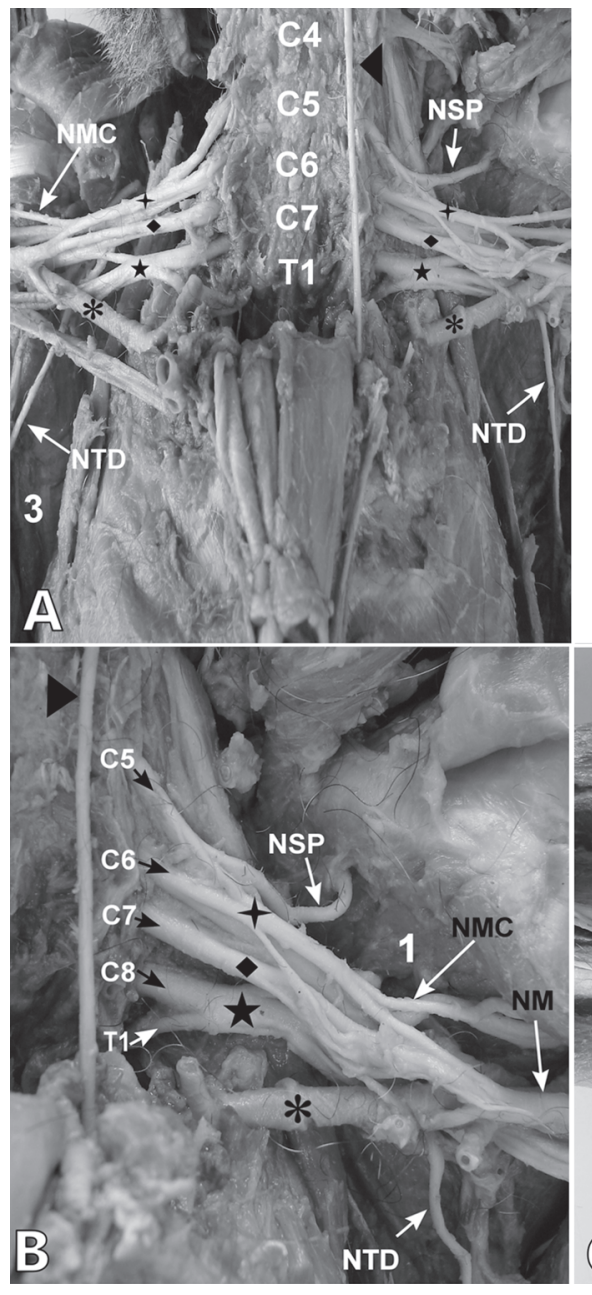

Fig.2. (A-D) Vista ventral do plexo braquial de macaco-barrigudo (Lagothrix lagothricha). (A) C4 a C7: quarta a sétima vértebras cervicais; T1: primeira vértebra torácica; Músculo: 3: grande dorsal; Nervos: NMC: musculocutâneo; NSP: supraescapular; NTD: toracodorsal. Artéria axilar $(\boldsymbol{*})$; tronco caudal ( $\star$ ). (B) C5 a C8: quinto a oitavo nervos espinhais cervicais; T1: primeiro nervo espinhal torácico; Troncos cranial $(+)$, médio $(\$)$ e caudal $(\star)$. Músculo: (1) subescapular. Nervos: NSP: supraescapular; NMC: musculocutâneo; NM: mediano; NTD: toracodorsal. Artéria axilar (*); tronco vagosimpático (4). (C) Músculos: (3) grande dorsal; (4 e 5) bíceps braquial; (6) coracobraquial; (9) tríceps braquial. Nervos: musculocutâneo (NMC); mediano (NM); toracodorsal (NTD). (D) Músculos: (4 e 5) bíceps braquial; (7) braquial; (8) tríceps braquial. Nervos: musculocutâneo (NMC); mediano (NM); ramos do nervo mediano (rNM); ligação de rNM com NMC (NM +NMC) com trajeto para o músculo bíceps braquial (5).

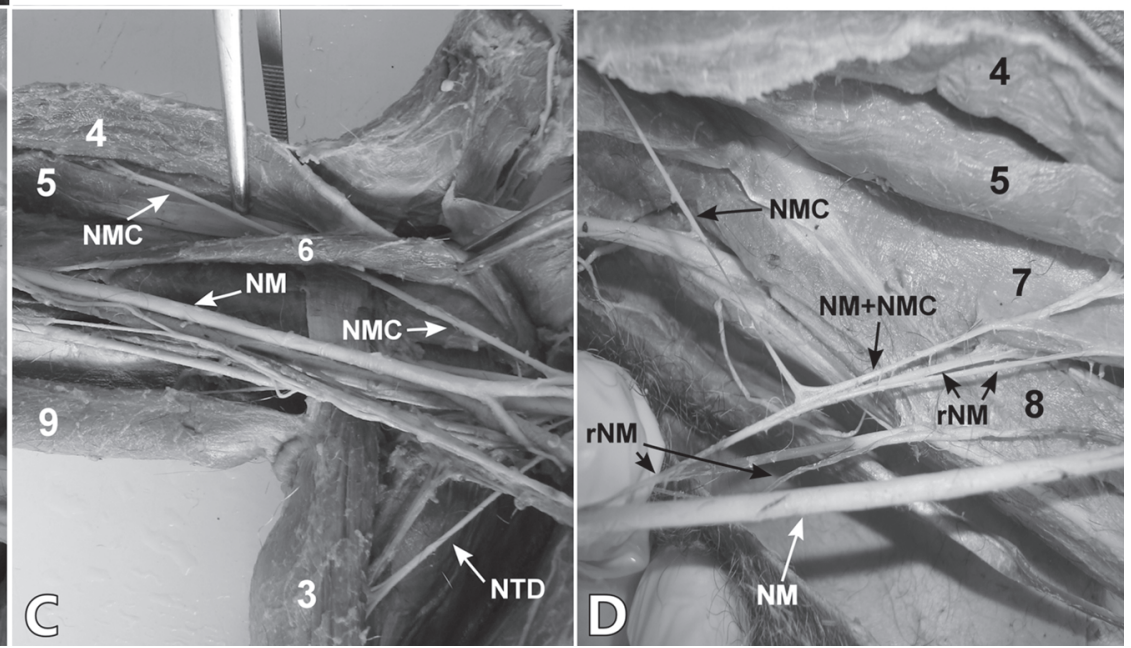




\section{DISCUSSÃO}

A origem e formação do plexo do macaco-barrigudo assemelharam-se com grande parte dos primatas já estudados. Brooks (1883) observou em espécies de Macaca a participação do $5^{\circ}, 6^{\circ}, 7^{\circ}$ e $8^{\circ}$ nervos cervicais e o $1^{\circ}$ nervo torácico, podendo receber contribuição considerável de C4. Em Lagothrix sp. o plexo braquial é formado pelas divisões dos três últimos nervos cervicais e o primeiro nervo torácico (Hill 1972), difereciando-se da maioria dos primatas que apresenta contribuição, mesmo que pequena, de C4 e/ou T2. Em macaco-de-cheiro (Saimiri sciureus) (Mizuno 1969a) e babuínos (Papio ursinus) foram observadas formações do plexo semelhantes ao macaco-barrigudo, podendo ocorrer contribuição de T2 (Booth et al. 1997). Kawashima et al. (2007) observaram na dissecação do plexo braquial de um orangotango (Pongo sp.) a participação de C5 a T1, podendo ocorrer também de C4.

A formação da alça axilar e a composição dos troncos corresponderam com o encontrado em macaco-prego-dacara-branca e babuínos (Mizuno 1969b, Booth et al. 1997) enquanto em Macaca, orangotango e gorila, a participação de C4 no tronco cranial e de T2 no tronco caudal (Sugiyama 1965, Koizumi \& Sakai 1995), diferiram do observado em macaco-barrigudo.

O tronco cranial formado por C5 e C6 assemelhou-se com o gálago (Galago senegalensis) (Kanagasuntheram \& Mahran 1960) e babuíno (Papio ursinus) (Booth et al. 1997), enquanto o tronco médio composto somente por $\mathrm{C}$, também foi observado em guenon (Cercopithecus) (Hill 1966); macaca (Macacus cyclopsis) (Sugiyama 1965); macacode-cheiro (Saimiri scirieus) (Mizuno 1969a); orangotango (Pongo sp.) e gorila (Gorilla sp.) (Koizumi \& Sakai 1995).

O tronco caudal assemelhou-se ao de macaca (Macacus cyclopsis) e macaco-prego (Cebus apella), porém nestes animais a presença de T2 na formação do plexo foi frequente (Sugiyama 1965, Ribeiro 2002).

A formação do plexo braquial de Lagothrix lagothricha equivaleu à mesma do macaco-prego-de-cara-branca (Cebus capucinus) (Mizuno 1969b). De acordo com Parada et al. (1989), durante o processo evolutivo a origem do plexo braquial deslocou-se cranialmente, alcançando $\mathrm{C} 4$ nos macacos e C3 em humanos. O plexo braquial recebe contribuição principal dos três últimos nervos espinhais cervicais e do primeiro torácico nos animais domésticos. A participação de ramos mais craniais, como C5 e C4, caracteriza um plexo pré-fixado como o observado em macacos do novo mundo e em Lagothrix que tem contribuição de C5 (Sugiyama 1965, Hill 1972).

No macaco-barrigudo a contribuição dos troncos para a formação dos nervos ocorreu em diferentes arranjos. Alguns nervos originaram-se exclusivamente de um tronco: os nervos supraescapular e o musculocutâneo a partir do tronco cranial; o nervo torácico longo do tronco médio e os nervos ulnar e o cutâneo medial do antebraço do tronco caudal. Em babuínos (Papio ursinus) o NSP e o NU também se originaram exclusivamente de um tronco, cranial e caudal, respectivamente, porém o NMC e o NTL não rece- beram contribuições exclusivas na sua formação (Booth et al. 1997). Em macaca (Macacus cyclopsis), o NSP, o NCMA e o NU surgiram de apenas um dos troncos (Sugiyama 1965), da mesma forma que Lagothrix lagothricha, enquanto todos os outros nervos derivados do plexo receberam contribuição de mais de um tronco.

Em chimpanzé (Pan sp.), orangotango (Pongo sp.) (Hepburn 1892), gálago (Galago senegalensis) (Kanagasuntheram \& Mahran 1960) e babuíno (Papio ursinus) (Booth et al. 1997), a origem e o território de inervação do nervo supraescapular foram iguais a de Lagothrix lagothricha. Hill (1972) relatou somente a contribuição de C6 em Macaca e, em L. lagothricha, principalmente por $\mathrm{C} 6$, podendo ser formado pelo tronco cranial (C5 e C6).

Em babuínos (Papio ursinus) a origem e o número de nervos subescapulares foram iguais aos de Lagothrix lagothricha, porém com inervação restrita ao músculo subescapular (Booth et al. 1997). Em gorila (Hepburn 1892) o número variou de 3 a 5 , com território de inervação semelhante ao do macaco-barrigudo.

Os nervos peitorais, em chimpanzé (Pan sp.), orangotango (Pongo sp.), gibão (Hylobates sp.) e gorila (Gorilla $\mathrm{sp}$.), originaram-se dos troncos cranial e médio e seguiram para os músculos peitorais maior e menor (Champneys 1975, Hepburn 1982), diferindo da origem em Lagothrix lagothricha, porém com o mesmo território de inervação. Em babuínos (Papio ursinus) sua origem foi do tronco médio com contribuição do NTL, direcionando-se para os músculos subclávio, peitorais maior e menor (Booth et al. 1997).

A origem do nervo musculocutâneo a partir tronco cranial assemelhou-se ao observado em guenon (Cercopithecus mona) (Hill 1966) e babuíno (Papio ursinus) (Booth et al. 1997). Em relação ao seu trajeto houve variação em relação a alguns primatas, a exemplo, macacoprego (Cebus apella) (Ribeiro 2002), babuínos (Papio ursinus) (Booth et al. 1997) e orangotango (Pongo sp.) (Kawashima et al. 2007). Todos diferiram de Lagothrix lagothricha que, semelhante ao homem (Chitra 2007) e ao chimpanzé (Pan sp.) (Champneys 1975, Koizumi \& Sakai 1995), o nervo musculocutâneo atravessou o músculo coracobraquial. Em gibão (Hylobates sp) o NMC após suprir o coracobraquial emitiu ramos para o bíceps e continuou seu curso no músculo braquial (Hepburn, 1892), corroborando com nossos achados. Em chimpanzé (Pan sp.), gibão (Hylobates sp.), macaco-aranha (Ateles sp), muriqui (Brachyteles) (Hill 1972), e macaco-prego (Cebus apella) (Aversi-Ferreira et al. 2005) os músculos inervados pelo NMC foram o coracobraquial, bíceps braquial e braquial à semelhança do macaco-barrigudo.

O nervo mediano apresentou um ramo comunicante com o NMC em diversas espécies de primatas condizente ao que foi observado em Lagothrix lagothricha no qual este ramo foi responsável pela contribuição do tronco cranial para a formação de nervo e da alça axilar. Em babuínos (Papio ursinus) o NM, o NU e o NCMA apresentaram origem de um tronco comum recebendo fibras de C8, T1 e T2 
e o NMC, que se originou de $\mathrm{C} 5$, $\mathrm{C} 6$ e C7, uniu-se ao NM de maneira que os troncos cranial e médio também contribuíram para a formação deste nervo além do tronco caudal, semelhante ao observado no macaco-barrigudo (Booth et al. 1997).

O nervo ulnar em gálago (Kanagasuntheram \& Mahran 1960) apresentou origem e composição iguais às de Lagothrix lagothricha. Em babuínos (Papio ursinus) o NU originou-se de um tronco comum com o NM e o NCMA recebendo fibras de C8, T1 e T2 (Booth et al. 1997). Mizuno (1969) afirmou que, em macaco-prego-de-cara-branca (Cebus capucinus), o NU e o NM não se separaram até se aproximarem da articulação do cotovelo.

O nervo radial originou-se de C6, tronco médio e C8 em gálago (Galago senegalensis) (Kanagasuntheram \& Mahran 1960) e em alguns espécimes de macaco-prego (Cebus apella) (Ribeiro 2002), diferente do encontrado em macaco-barrigudo. Em babuínos (Papio ursinus) todos os nervos espinhais do plexo braquial contribuíram diretamente na origem deste nervo (Booth et al. 1997), indo ao encontro do observado neste trabalho. Hill (1966) descreveu em Cercopithecus a inervação do músculo tríceps braquial e em muriqui (Brachyteles) houve a emissão de ramos cutâneos para o músculo deltóide.

O nervo axilar, em alguns primatas, derivou dos troncos cranial e médio e contribuiu para a formação do NR (Hill 1972, Ribeiro 2002). Em chimpanzé (Troglotydes niger), anubis (Cynocephalus anubis) (Champneys 1975) e em babuínos (Papio ursinus) (Booth et al. 1997) houve contribuição dos troncos cranial e médio, com território de inervação semelhante ao do Lagothrix lagothricha.

O nervo torácico longo em gorila (Gorilla sp.), orangotango (Pongo sp.) e chimpanzé (Pan sp.) originou-se do tronco cranial (Hepburn 1892, Champneys 1975), em anubis (Cynocephalus anubis) de C6, tronco médio e, ocasionalmente, C8 (Champneys 1975) e em Cercopithecus houve a contribuição de $\mathrm{C} 6$, podendo receber de $\mathrm{C} 5$ e do tronco médio (Hill 1966). Em macaco-prego (Cebus apella), Ribeiro (2002) observou a participação dos troncos cranial e médio. No Lagothrix lagothricha a origem do NTL diferiu dos outros primatas estudados, pois se originou de apenas um dos troncos e seu território de inervação supriu o músculo serrátil ventral, conforme descrito na maioria dos primatas (Hill 1972, Hepburn 1982, Booth et al. 1997, Ribeiro 2002).

O nervo toracodorsal, conforme descrito por Hill (1972), em Lagothrix sp., dirigiu-se para o músculo grande dorsal, com origem apenas do oitavo nervo cervical. Em babuínos (Papio ursinus) (Booth et al. 1997) sua origem foi descrita a partir do tronco comum com o NR, recebendo contribuição de todos os nervos espinhais que formaram o plexo braquial e o território de inervação correspondeu ao músculo grande dorsal, semelhante ao encontrado em Lagothrix lagothricha.

A origem do nervo cutâneo medial do antebraço em macaco-barrigudo correspondeu à mesma origem descrita no macaco-prego (Cebus apella) por Hill (1972), contudo, em Cercopithecus este nervo originou-se somente de C8 (Hill 1966), apresentando, porém, o mesmo território de inervação. Em babuínos (Papio ursinus) a origem foi a partir do tronco comum do NM e NU e o território de inervação equivaleu à porção medial da pele (Booth et al. 1997), diferindo do Lagothrix lagothricha.

\section{CONCLUSÕES}

O plexo braquial de Lagothrix lagothricha originou-se dos nervos espinhais C5 a T1 constituindo os troncos cranial, médio e caudal.

Os nervos periféricos derivados dos troncos assemeIharam na origem, trajeto e no território de inervação com os plexos de outros primatas, com exceção do nervo musculocutâneo que atravessou o músculo coracobraquial.

Na formação dos nervos do plexo houve a participação de um, dois ou dos três troncos nervosos.

\section{REFERÊNCIAS}

Auricchio P. 1995. Primatas do Brasil. Terra Brasilis, São Paulo. 168p. Aversi-Ferreira T., Silva M.S.L., Paula J.P., Silva L.G. \& Silva N.P. 2005. Anatomia comparativa dos nervos do braço de Cebus apella: descrição do músculo dorsoepitroclear. Acta Sci. Biol. Sci. 27(3):291296.

Booth K.K., Baloyi F.M. \& Lukhele O.M. 1997. The brachial plexus in the Chacma baboon (Papio ursinus). J. Med. Primatol. 26:196-203.

Brooks W.T. 1883. The brachial plexus of the Macaque monkey and its analogy with that of man. J. Anat. Physiol. 17(3):329-332.

Champneys F. 1975. On the muscles and nerve of a Chimpanzee (Troglodytes niger) and Cynocephalus anubis. J. Anat. Phys. 6(1):176-211.

Chiarelli A.B. 1972. Taxonomic Atlas of Living Primates. Academic Press, London. 63p.

Chitra R. 2007. Various types of intercommunications between musculocutaneous and median nerves: An analytical study. Ann. Indian Acad. Neurol. 10:100-104.

Hepburn D. 1892. The comparative anatomy of the muscles and nerves of the superior and inferior extremities of the anthropoid apes. Part I. J. Anat. Physiol. 26(2):149-186.

Hill W.C.O. 1972. Primates: Comparative anatomy and taxonomy. V. Cebidae: Part B. Edinburgh University Press, Edinburgh. 537p.

Hill W.C.O. 1966. Primates: Comparative anatomy and taxonomy. VI. Catarrhini: Cercopithecoidea-Cercopithecinae. Edinburgh University Press, Edinburgh. 757p.

International Committee on Veterinary Gross Anatomical Nomenclature 2005. Nomina Anatomica Veterinaria. $5^{\text {th }}$ ed. Hannover $166 p$.

Johnson S.E. \& Shapiro L.J. 1998. Positional behavior and vertebral morphology in Atelines and Cebines. Am. J. Phys. Anthropol. 105:333354.

Kanagasuntheram R. \& Mahran Z.Y. 1960. Observations on the nervous system of the lesser bush baby (Galago senegalensis senegalensis). J. Anat. 94(4):512-527.

Kawashima T., Yoshitomi S. \& Sasaki H. 2007. Nerve fibre tracing of branches to the coracobrachialis muscle in a Bornean orangutan (Pongo pygmaeus pygmaeus). Anat. Histol. Embryol. 36(1):19-23.

Koizumi M. \& Sakai T. 1995. The nerve supply to coracobrachialis in apes. J. Anat. 186(2):395-403.

Mizuno N. 1969a. The brachial plexus in the Squirrel Monkey (Saimiri sciureus). Primates 10:19-35. 
Mizuno N. 1969b. The brachial plexus of a Capuchin Monkey (Cebus capucinus). Primates 10:37-40.

Moura C.E.B., Albuquerque J.F.G., Magalhães M.S., Silva N.B., Oliveira M.F. \& Papa P.C. 2007. Análise comparativa da origem do plexo braquial de catetos (Tayassu tajacu). Pesq. Vet. Bras. 27(9):357362.

Napier J.P. \& Napier P.H. 1967. A handbook of Living Primates: Morphology, ecology and behaviour of nonhuman primates. Academic Press, New York. 456p.

Oliveira M.F., Miglino M.A., Passipieri M., Teixeira D.G., Moura C.E.B., Martins D.S., Benedicto H., Thomaz J.M. \& Ambrósio C.E. 2003. Anatomia do plexo braquial do macaco Gibão (Hylobates sp.). Int. J. Morphol. 21(1):59.

Parada H., Pineda U.H., Lagunas E.M. \& Vidal H.A. 1989.Variaciones anatômicas de las ramas raquídeas que constituyen los troncos de origen del plexo braquial. Anales Anat. Normal 7:32-36. (Apud Moura et al. 2007)
Reis N.R., Peracchi A.L., Pedro W.A. \& Lima I.P. 2006. Mamíferos do Brasil. Nélio R. dos Reis, Londrina. 437p.

Ribeiro A.R. 2002. Estudo anatômico do plexo braquial do macaco Cebus apella: origem, composição e nervos resultantes. Dissertação de Mestrado em Anatomia dos Animais Domésticos, Faculdade de Medicina Veterinária e Zootecnia, USP, São Paulo. 146p.

Rylands A. \& Members of the Primate Specialist Group 2000. Lagothrix lagothricha. In: IUCN 2007. Red List of Threatened Species. <www.iucnredlist.org> Downloaded on 13 April 2008.

Sugiyama T. 1965. On the plexus brachialis of Macacus cyclopsis. Acta Med. Nagasaki. 9(3):65-68.

Youlatos D. 2000. Functional anatomy of forelimb muscles in Guianan Atelines (Platyrrhini: Primates). Annales des Sciences Naturalles 21(4):137-151.

Ziemer L.K. 1978. Functional morphology of forelimb joints in the woolly monkey Lagothrix lagothricha. Contrib. Primatol. 14:1-130. (Resumo) 